\title{
Evaluations of auto-focusing methods under a microscopic imaging modality for metaphase chromosome image analysis
}

\author{
Yuchen Qiu ${ }^{\mathrm{a}}$, Xiaodong Chen ${ }^{\mathrm{a}, \mathrm{b}}$, Yuhua $\mathrm{Li}^{\mathrm{a}}$, Wei R. Chen ${ }^{\mathrm{c}}$, Bin Zheng ${ }^{\mathrm{d}}$, Shibo Lie and Hong $\mathrm{Liu}^{\mathrm{a}, *}$ \\ ${ }^{\mathrm{a}}$ Center for Bioengineering and School of Electrical and Computer Engineering, University of Oklahoma, \\ Norman, OK, USA \\ ${ }^{\mathrm{b}}$ College of Precision Instrument and Opto-Electronics Engineering, Tianjin University, Tianjin, China \\ ${ }^{\mathrm{c}}$ Department of Engineering and Physics, University of Central Oklahoma, Edmond, OK, USA \\ ${ }^{\mathrm{d}}$ Department of Radiology, University of Pittsburgh, Pittsburgh, PA, USA \\ ${ }^{\mathrm{e}}$ Department of Pediatrics, University of Oklahoma Health Sciences Center, Oklahoma City, OK, USA
}

\begin{abstract}
.
BACKGROUND: Auto-focusing is an important operation in high throughput imaging scanning. Although many auto-focusing methods have been developed and tested for a variety of imaging modalities, few investigations have been performed on the selection of an optimal auto-focusing method that is suitable for the pathological metaphase chromosome analysis under a high resolution scanning microscopic system.

OBJECTIVE: The purpose of this study is to investigate and identify an optimal auto-focusing method for the pathological metaphase chromosome analysis.

METHODS: In this study, five auto-focusing methods were applied and tested using metaphase chromosome images acquired from bone marrow and blood specimens. These methods were assessed by measuring a number of indices including execution time, accuracy, number of false maxima, and full width at half maximum (FWHM).

RESULTS: For the specific condition investigated in this study, the results showed that the Brenner gradient and threshold pixel counting methods were the optimal methods for acquiring high quality metaphase chromosome images from the bone marrow and blood specimens, respectively.

CONCLUSIONS: Selecting an optimal auto-focusing method depends on the specific clinical tasks. This study also provides useful information for the design and implementation of the high throughput microscopic image scanning systems in the future digital pathology.
\end{abstract}

Keywords: Metaphase chromosome, autofocus technique

\section{Introduction}

High throughput microscopy is an important technique for the diagnosis and treatment of genetic related diseases [1-4]. To make this technology clinically

${ }^{*}$ Corresponding author: Hong Liu, Center for Bioengineering and School of Electrical and Computer Engineering, University of Oklahoma, 101 David L Boren Blvd, Norman, OK 73019, USA. Tel.: +1 405325 4286; Fax: +1 405325 7066; E-mail: liu@ou.edu. acceptable, it is critically important to efficiently obtain the in-focused high resolution microscopic images, as the blurred images may directly affect the diagnostic accuracy. Therefore, the autofocus technique is required for the high throughput microscopic system in the clinical practice.

During the last twenty years, substantial research efforts have been devoted to the development of reliable autofocus techniques for automated digital 
microscopes and other optical imaging applications [5-11]. Although it has been well known that the performance of the autofocus operation heavily depends on the selection of the autofocus function [12-15], a focusing function that performs well for the digital camera might not be selected as the optimal function for the digital scanning microscope [12]. Recently, some researchers have investigated and compared several different autofocus techniques for scanning a number of specific pathological specimens acquired from blood smear, pap smear, tuberculosis, or fluorescent samples [14, 16-18]. However, these researches are not specifically designed for the clinical specimens (i.e. blood or bone marrow) used in the pathological metaphase chromosome analysis.

In this study, we investigated and compared a number of different auto-focusing methods when they were applied to acquire metaphase chromosome images from bone marrow and blood specimens. The optimal auto-focusing method is selected and recommended based on the experimental results. The details of our experimental methods and results are presented as follows.

\section{Materials and methods}

In microscopic imaging, the obtained images will become fuzzy with decreased contrast and edge sharpness when the imaged objects (e.g. metaphase chromosomes) are located outside of the focal plane. The image contrast can be estimated by several autofocus functions. Therefore, the in-focused position of the imaged objects can be determined by searching for the maximal image contrast value.

In this investigation, a number of five different autofocus functions were evaluated using the metaphase chromosome images acquired from the bone marrow and blood specimens. All the experiments were performed on a prototype microscopic image scanning system previously developed in our medical image laboratory [19]. The specimens were prepared based on the standard clinical procedure.

During the experiment, we selected and tested a number of the autofocus functions aiming to obtain the high contrast images with maximum sharpness of chromosome band patterns. The published autofocus functions can be grouped into several classes including but not limited to: i) image gradient [8, 13-15], ii) histogram or contrast [13-15], iii) statistical mea- surement (e.g. correlation) [9], iv) wavelet transform $[15,20,21]$, and v) discrete cosine transform [11]. In this study, we tested five typical methods selected from group i), ii) and iii), which are Brenner gradient, histogram range, threshold pixel counting, Vollath F5, and variance $[8-10,12,13]$. These methods have been used for a variety of biomedical specimens including fluorescent sample, blood smear, pop smear, and tuberculosis [14, 16-18]. The concepts of these functions are briefly described as follows:

1) Brenner gradient: For each pixel on the captured image, this method calculates the square of the difference between its two neighbors, and then adds them together using the following equation [8]:

$$
F=\sum_{x, y}(i(x+1, y)-i(x-1, y))^{2},
$$

with $|i(x+1, y)-i(i-1, y)|>\alpha$, where $\mathrm{i}(\mathrm{x}$, $y)$ is the intensity at pixel $(x, y), \alpha$ is the threshold of the intensity difference.

2) Histogram range: It is defined as the difference between the maximum and minimum pixel intensities measured on the acquired image. Let $\mathrm{N}_{\mathrm{k}}$ be the number of pixels with intensity $\mathrm{k}(0 \leq \mathrm{k} \leq 255$ for the 256 level grayscale images), and the histogram range can be written as [13]:

$$
F=\max \left(k \mid N_{k} \neq 0\right)-\min \left(k \mid N_{k} \neq 0\right)
$$

3) Threshold pixel counting: It is defined as the number of pixels whose intensity is lower than a predetermined intensity (or grayscale) threshold [12]:

$$
F=\Sigma_{x, y} \operatorname{sign}[i(x, y), t h],
$$

where the sign function is 1 if the pixel intensity is below the threshold and 0 otherwise.

4) Vollath F5: It is defined on the basis of the standard deviation function [9]:

$$
F=\Sigma_{x, y} i(x, y) \cdot i(x+1, y)-M N \bar{i}^{2},
$$

where $\mathrm{M}, \mathrm{N}$ are the length and width of the image, respectively.

5) Variance: For each pixel of the image, this method computes the square of difference between pixel intensity and the average pixel value of the image, and then adds them together for the final value $[10,12,14]$ :

$$
F=\Sigma_{x, y}(i(x, y)-\bar{i})^{2}
$$



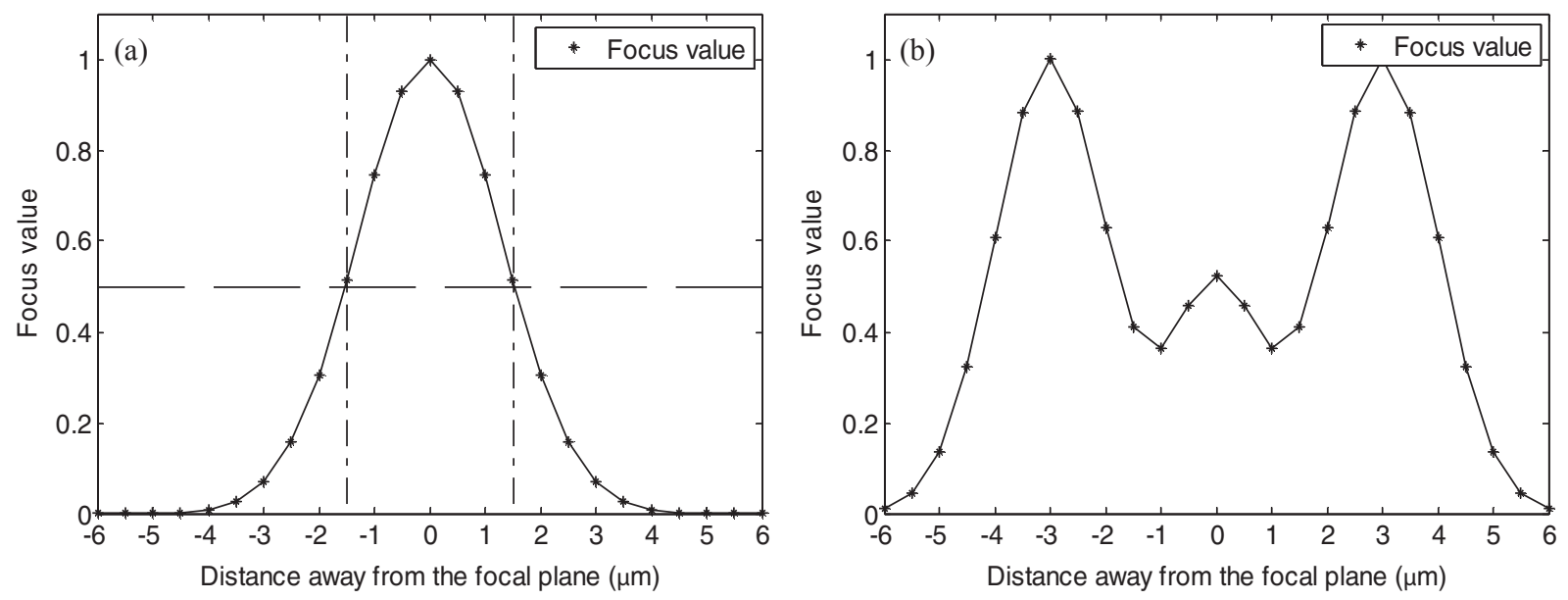

Fig. 1. Examples of an ideal focus curve (a) and a failed focus curve (b). The calculated focus value is plotted as a function of focusing position. The ideal focus curve in (a) is approximated by Gaussian function. It has only one maximum value, which corresponds to the focal plane ( $0 \mu \mathrm{m}$ ). The focus value decreases when the cell moves away. The range where the focus value is above $50 \%$ of the maximum is defined as full width at the half maximum (FWHM). Image (b) is a failed autofocus curve. The focus curve has two maximal values, thus the focal position cannot be located.

In the above five functions, the focus value $\mathrm{F}$ is an estimation of image contrast. Since image contrast is smaller than 1, the computed focus values are normalized for each metaphase chromosome cell.

In order to evaluate the performance of the above five different autofocus functions, the off-line (static) evaluation method was applied in this investigation, which is widely accepted as a standard method in this field [12-15, 17, 18]. This method assesses the autofocus functions using the previously captured chromosome images. In this study, chromosome images were obtained from blood and bone marrow specimens. For each specimen, a number of twenty metaphase chromosome cells were selected and used. For each cell, the focal position was first visually determined by the trained researchers. Then, a number of 25 images were captured for each cell by moving the scanning stage up and down in a range from $+6 \mu \mathrm{m}$ to $-6 \mu \mathrm{m}$, with a step of $0.5 \mu \mathrm{m}$. To acquire clinically acceptable images, a $100 \times$ oil immersion objective lens is used in the experiments.

The performance of using each of these autofocus functions was then assessed based on the acquired images. The autofocus function was applied on each captured image and the focus value was calculated. The computed focus value was curved as a function of focusing positions. For a typical autofocus curve as illustrated Fig. 1(a), it has only one maximum, and the focusing position corresponding to the maximum value is determined as the in-focused position.
In order to assess the autofocus function, four evaluation criteria were applied in this study, including execution time, focusing accuracy, number of false maxima, and full width at the half maximum (FWHM). These measuring parameters are described as follows $[14,15,18]$ :

1) Execution time: The time used to compute the autofocus value for each captured image.

2) Focusing accuracy: The difference between the visually determined and automatically determined focal positions. In this study, the visually determined position is calibrated at the central position $(0 \mu \mathrm{m})$.

3) Number of false maxima: False maximum is defined as the failed autofocus curve, as illustrated in Fig. 2(b). In this case, the in-focused position cannot be determined from the curve.

4) FWHM: As shown in Fig. 1(a), the autofocus value decreases vastly when the targeted cell is moved away from the in-focused plane. FWHM is the range where the autofocus function value reduces to $50 \%$ of the maximum.

The evaluation results were tabulated for comparison and analysis. Among the applied criteria, the number of false maxima was first considered, as it directly reflects the efficacy of the autofocus functions. The execution time was then compared, which reflects the efficiency of the operation. The FWHM was analyzed next. The standard of the FWHM is related to the 

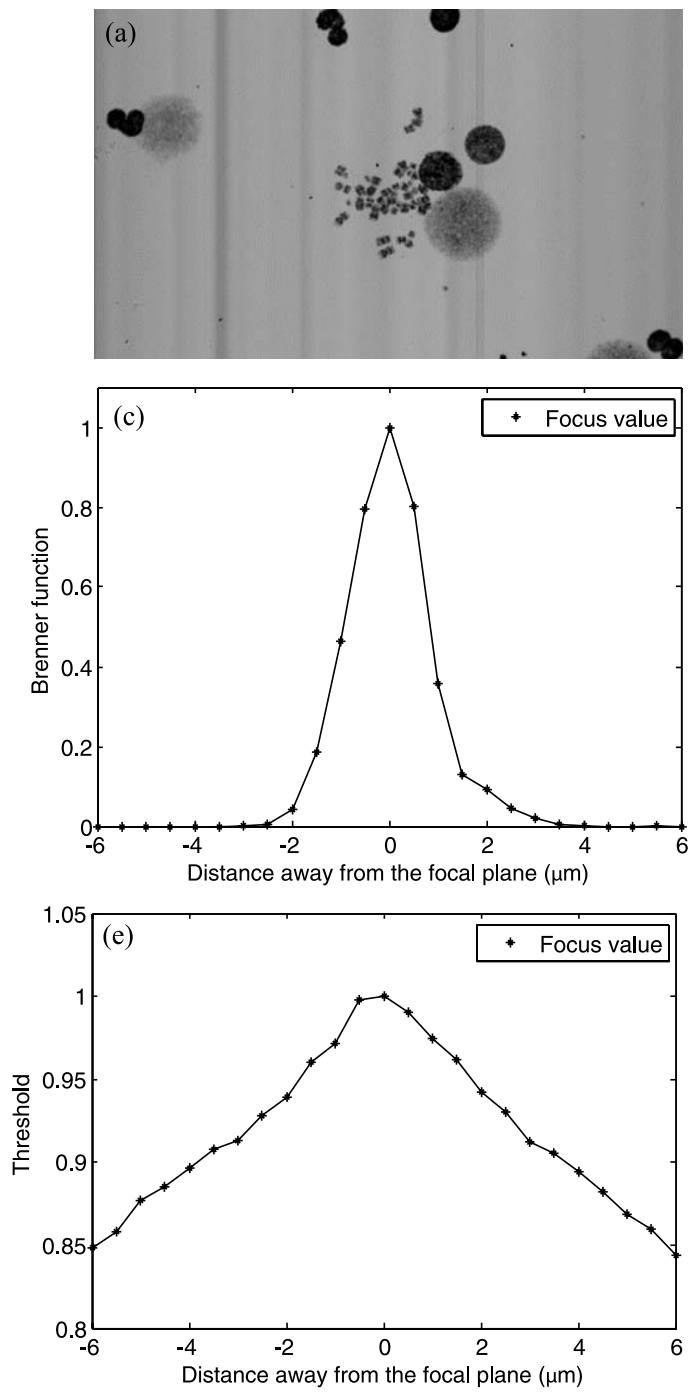
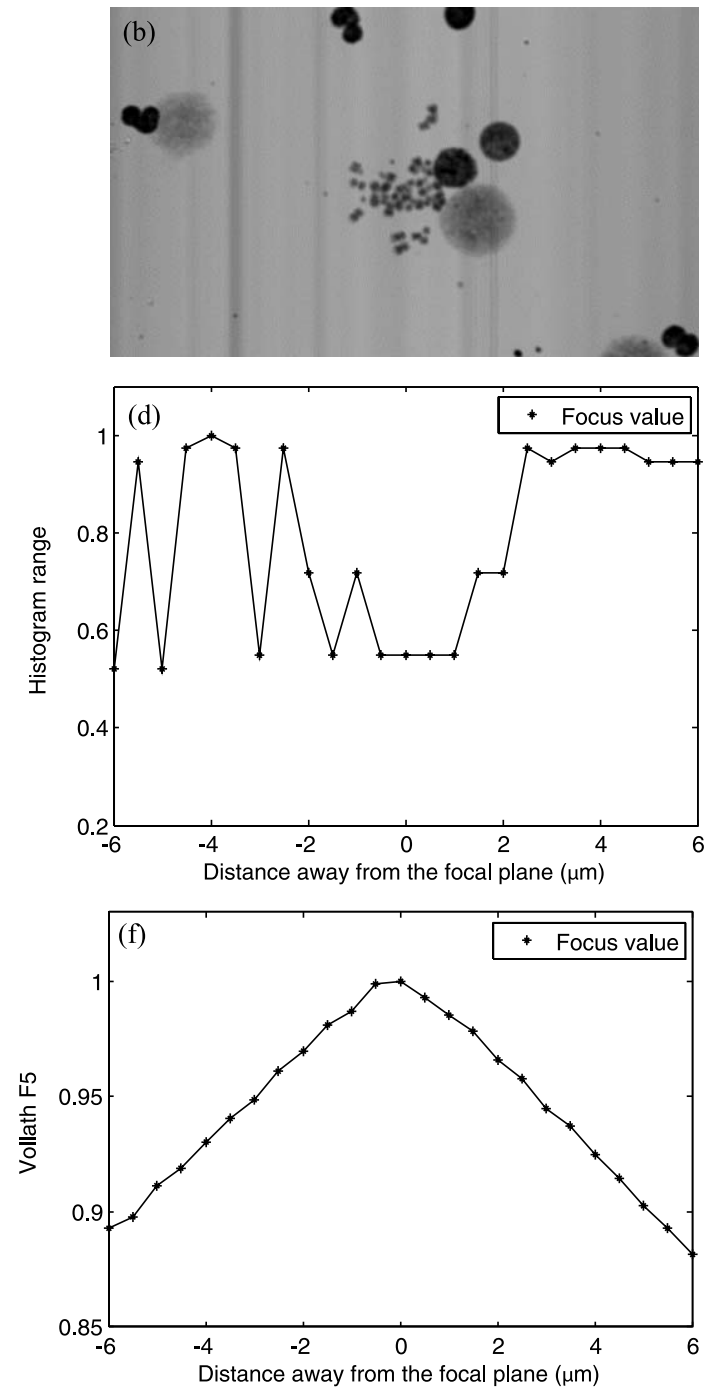

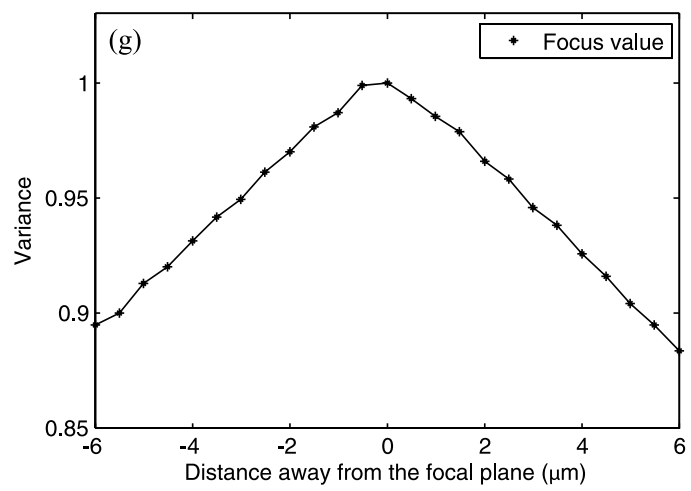

Fig. 2. An example of autofocus functions performed on micorsopic images of a pathlogical cell acquried from bone marrow sample. Image (a) and (b) are captured at in-focused position, and $3 \mu \mathrm{m}$ away from the focal plane, respectively. The image (b) is blurred as compared to the in-focused image in (a). The Brenner function (c), threshold pixel counting (e), Vollath F5 (f), and variance (g) successfully select the focal position. Histogram range (d), however, fails to locate the focal position. 
system depth of field (DOF), as the ideal focus curve can be approximated by the DOF contrast curve. The DOF is defined as the range where the measured contrast is larger than $80 \%$ of the maximum [22]. Under the experimental conditions (using $100 \times$ oil immersion objective lens), the measured DOF is $1.8 \mu \mathrm{m}$. Thus, the 'ideal' FWHM will be approximately $3.0 \mu \mathrm{m}$, as shown in Fig. 1(a). The focusing accuracy will not be used for comparison if it is within the system's DOF. All autofocus algorithms were assessed using a personal computer equipped with an Intel i3 $2.4 \mathrm{G} \mathrm{Hz}$ dual core processor with 4G RAM using the MATLAB R2011 software application.

\section{Results}

Figure 2 demonstrates an example of the autofocus functions. Figure 2 (a) and (b) demonstrate two images that were separately acquired at the in-focus plane and $3 \mu \mathrm{m}$ away. Among these two images, Fig. 2 (b) is obviously blurred. Figure 2 (c)-(g) illustrate the results of the five different autofocus functions. It reveals that the Brenner function, threshold, Vollath F5 and variance methods can effectively locate the focal position, as the calculated value reaches the maximum around the focal plane $(0 \mu \mathrm{m})$. The Brenner function deceases faster than the other three methods when the cell is moved away from the in-focused plane. Histogram range, however, fails to find the focal plane. The range value varies at different positions and no peak value can be found.

In the high throughput scanning microscope, the captured image is very large $(3488 \times 2048,3488$ pixels in $\mathrm{x}$ direction and 2048 pixels in y direction) and also contains interphase cells. As compared to the chromosome bands, the size of the interphase nuclei is larger, thus the spatial frequency is lower. According to the Fourier optics theory, the contrast of high spatial frequency regions decreases more significantly than the low frequency regions when the cell is moved away from the focal position [23]. Therefore, this kind of system requires that the autofocus function can extract the useful high frequency components from the obtained image. Among all the five selected methods, Brenner function performs better than the others, as the difference operator can extract the high frequency information while discarding the others. The threshold pixel counting, Vollath F5, and variance methods can somehow extract the useful high frequency information. The pixel intensity variance of in-focused images is larger than the off focused images. This variance can be reflected by calculating the image variance (variance method), standard deviation (Vollath F5), or counting pixels with very low grayscale (threshold pixel counting). Histogram range method, however, cannot distinguish the high and low frequency components, as the range are mainly determined by the low frequency components in this situation.

Tables 1 and 2 summarize the statistical results of applying the five autofocus functions on the bone marrow and blood samples. It also demonstrates that Brenner function and threshold pixel count methods are superior to the others. Both these two methods can successfully locate the focal position with high reliability. The threshold pixel counting method has one false maximum when it is performed on the bone marrow samples. For assessing efficiency, however, the threshold method is much higher than the Brenner function. It takes about 15 seconds for the Brenner function to process a single image, while threshold method only needs about 0.2 second. This is due to the high computing complexity of the Brenner gradient method. The FWHM of the Brenner method is approximately $1.5 \mu \mathrm{m}$, while the FWHM of the threshold method is larger than $12.5 \mu \mathrm{m}$. As compared to the threshold method, the FWHM of the Brenner function (approximately $1.5 \mu \mathrm{m}$ ) is closer to the ideal FWHM,

Table 1

Results of the evaluation of autofocus functions for bone marrow specimen

\begin{tabular}{lcccc}
\hline & $\begin{array}{c}\text { Executing time } \\
(\text { second })\end{array}$ & Accuracy $(\mu \mathrm{m})$ & $\begin{array}{c}\text { Number of } \\
\text { false maxima }\end{array}$ & $\begin{array}{c}\text { Full width at } \\
\text { half maximum }(\mu \mathrm{m})\end{array}$ \\
\hline Brenner function & $14.7125 \pm 0.6406(3)$ & $0.2500 \pm 0.2565(1)$ & $0(1)$ & $1.6344 \pm 0.1630(1)$ \\
Histogram range & $0.1411 \pm 0.0066(2)$ & $1.3000 \pm 0.6708(5)$ & $15(5)$ & $\geq 12.5(2)$ \\
Threshold & $0.1383 \pm 0.0033(1)$ & $0.2895 \pm 0.2536(4)$ & $1(2)$ & $\geq 12.5(2)$ \\
Vollath F5 & $34.7059 \pm 3.0202(4)$ & $0.2632 \pm 0.2565(2)$ & $1(2)$ & $\geq 12.5(2)$ \\
Variance & $35.0119 \pm 0.8372(5)$ & $0.2632 \pm 0.2565(2)$ & $1(2)$ & $\geq 12.5(2)$ \\
\hline
\end{tabular}

Note: Rank is illustrated in the parentheses. 
Table 2

Results of the evaluation of autofocus functions for blood specimen

\begin{tabular}{lrccc}
\hline & $\begin{array}{c}\text { Executing time } \\
\text { (second) }\end{array}$ & Accuracy $(\mu \mathrm{m})$ & $\begin{array}{c}\text { Number of } \\
\text { false maxima }\end{array}$ & $\begin{array}{c}\text { Full width at } \\
\text { half maximum }(\mu \mathrm{m})\end{array}$ \\
\hline Brenner function & $15.3441 \pm 0.4044(3)$ & $0.2250 \pm 0.2552(2)$ & $0(1)$ & $1.3404 \pm 0.6354(1)$ \\
Histogram range & $0.1435 \pm 0.0099(2)$ & $1.1818 \pm 0.7833(5)$ & $9(5)$ & $\geq 12.5(2)$ \\
Threshold & $0.1400 \pm 0.0064(1)$ & $0.2000 \pm 0.2513(1)$ & $0(1)$ & $\geq 12.5(2)$ \\
Vollath F5 & $32.0297 \pm 0.6356(4)$ & $0.2500 \pm 0.3035(3)$ & $0(1)$ & $\geq 12.5(2)$ \\
Variance & $34.5461 \pm 0.2625(5)$ & $0.2500 \pm 0.3035(3)$ & $0(1)$ & $\geq 12.5(2)$ \\
\hline
\end{tabular}

Note: Rank is illustrated in the parentheses.

which shows that the Brenner function is more sensitive to the change of focusing position. Thus, it can search the focal plane more reliably than the threshold method. The accuracies of both these two methods are within the system's DOF.

Blood and bone marrow samples have different optimal autofocus functions. In clinical application, one or two autofocus false maxima is acceptable when scanning the blood specimens, as one slide usually contains $30-50$ useful cells and clinicians only need 3-5 cells to make the diagnosis. Therefore, the threshold pixel counting method is the optimal selection, because the accuracy and robustness of this method is satisfactory but it has much higher efficiency than the Brenner function method.

For the bone marrow specimen, however, one slide only contains 5-6 useful analyzable metaphase cells. In order to collect enough (20) cells for diagnosis, clinicians need to screen 3-5 slides. Furthermore, even in in-focused state, the image quality of bone marrow cells is not as good as the blood cells, which may affect the autofocus operation. Thus, scanning of bone marrow slides requires very high reliability. On the other hand, executing time of the Brenner gradient is highly dependent on the computing environment. The executing time can be significantly reduced by utilizing a high efficiency programming language such as $\mathrm{C} / \mathrm{C}++$ under the environment of a high performance workstation. Therefore, Brenner function is the optimal solution for the bone marrow slide, as it has high accuracy and robustness to the useless information, especially when using the images with decreased quality.

\section{Discussion}

Metaphase chromosome karyotyping of pathological specimens is a widely used technique for the diagnosis of genetic diseases. In the hospital, clinicians need to carefully examine the number or morphology of the chromosome bands, to determine whether the case is abnormal or not $[1,3,4,24,25]$. Therefore, during the image acquisition, it is critically important to ensure that the band sharpness is adequate for diagnosis, as the off-focused bands in the captured images might lead to false positive or false negative diagnostic results.

In order to keep the adequate band sharpness in microscopic images, autofocus techniques are necessary for the automatic or semi-automatic scanning microscopes, especially the high throughput scanning systems [26, 27]. The autofocus technique can be divided into autofocus function and searching algorithms. Since the performance of autofocus functions varies in different applications, the autofocus function must be carefully selected to achieve the satisfactory or optimal results. Although several studies have been reported on selecting the optimal autofocus function for some specimens, such as pap smear or tuberculosis, little effort has been done on how to select the "best" autofocus function for the metaphase chromosome images acquired from different pathological specimens (i.e. bone marrow or blood) in high resolution imaging environments [14, 16-18].

In this study, five autofocus functions were tested and compared on metaphase chromosome images obtained from bone marrow and blood specimens. Four different criteria were used for the evaluation. The results show that the Brenner gradient and threshold pixel counting are superior to the others. To achieve the optimal performance, Brenner gradient and threshold pixel counting methods are suggested for the bone marrow and blood sample scanning, respectively.

However, this is a preliminary study with several limitations. First, only blood and bone marrow samples were used in this investigation. Some other specimens, such as amniotic fluid, product of conception (POC), which are also widely used in clinical practice, 
were not tested. Second, we only selected five different autofocus functions in this study. Some recently developed functions were not considered [5, 28-30]. Third, the selected optimal autofocus methods have not been actually performed for realistic imaging scanning. Hence, a more comprehensive study is underway, which may help eventually optimize high throughput microscopic image scanning system in the future clinical practice.

\section{Acknowledgments}

This research is supported in part by grants RO1 CA115320 and CA136700 from the National Cancer Institute, National Institutes of Health, U.S.A.; and supported in part by a grant from the University of Oklahoma Charles and Peggy Stephenson Cancer Center funded by the Oklahoma Tobacco Settlement Endowment Trust. The authors would like to acknowledge the support of Charles and Jean Smith Chair endowment fund as well.

\section{References}

[1] P.C. Nowell and D.A. Hungerford, Minute chromosome in human chronic granulocytic leukemia, Science 132 (1960), 1497.

[2] J. Piper, E. Granum, D. Rutovitz and H. Ruttledge, Automation of chromosome analysis, Signal Processing 2 (1980), 203-221.

[3] G.M. Hampton, A.A. Larson, R.N. Baergen, R.L. Sommers, S. Kern and W.K. Cavenee, Simultaneous assessment of loss of heterozygosity at multiple microsatellite loci using semiautomated fluorescence-based detection: Subregional mapping of chromosome 4 in cervical carcinoma, Proceedings of the National Academy of Sciences of the United States of America 93 (1996), 6704-6709.

[4] J.H. Tjio and A. Levan, The chromosome number of man, Hereditas 42 (1956), 1-6.

[5] S. Yazdanfar, K.B. Kenny, K. Tasimi, A.D. Corwin, E.L. Dixon and R.J. Filkins, Simple and robust image-based autofocusing for digital microscopy, Optics Express 16 (2008), 8670-8677.

[6] S. Yousefi, M. Rahman and N. Kehtarnavaz, A new auto-focus sharpness function for digital and smart-phone cameras, IEEE Transactions on Consumer Electronics 57 (2011), 1003-1009.

[7] M.E. Bravo-Zanoguera, C.A. Laris, L.K. Nguyen, M. Oliva and J.H. Price, Dynamic autofocus for continuous-scanning time-delay-and-integration image acquisition in automated microscopy, Journal of Biomedical Optics 12 (2007), 034011-034016.

[8] J.F. Brenner, B.S. Dew, J.B. Horton, T. King, P.W. Neurath and W.D. Selles, Automated microscope for cytologic research - preliminary evaluation, Journal of Histochemistry \& Cytochemistry 24 (1976), 100-111.
[9] D. Vollath, The influence of the scene parameters and of noise on the behavior of automatic focusing algorithms, Journal of Microscopy 151 (1988), 133-146.

[10] T.T.E. Yeo and S.H. Ong, Jayasooriah, and R. Sinniah, Autofocusing for tissue microscopy, Image and Vision Computing 11 (1993), 629-639.

[11] J. Jeon, J. Lee and J. Paik, Robust focus measure for unsupervised auto-focusing based on optimum discrete cosine transform coefficients, IEEE Transactions on Consumer Electronics 57 (2011), 1-5.

[12] F.C.A. Groen, I.T. Young and G. Ligthart, A comparison of different focus functions for use in autofocus algorithms, Cytometry 6 (1985), 81-91.

[13] L. Firestone, K. Cook, K. Culp, N. Talsania and K. Preston, Comparison of autofocus methods for automated microscopy Cytometry 12 (1991), 195-206.

[14] A. Santos, C.O. De Solorzano, J.J. Vaquero, J.M. Pena, N. Malpica and F. Del Pozo, Evaluation of autofocus functions in molecular cytogenetic analysis, Journal of Microscopy 188 (1997), 264-272.

[15] Y. Sun, S. Duthaler and B.J. Nelson, Autofocusing in computer microscopy: Selecting the optimal focus algorithm, Microscopy Research and Technique 65 (2004), 139-149.

[16] X.Y. Liu, W.H. Wang and Y. Sun, Dynamic evaluation of autofocusing for automated microscopic analysis of blood smear and pap smear, Journal of Microscopy 227 (2007), 15-23.

[17] O.A. Osibote, R. Dendere, S. Krishnan and T.S. Douglas, Automated focusing in bright-field microscopy for tuberculosis detection, Journal of Microscopy 240 (2010), 155-163.

[18] J.M. Mateos-Perez, R. Redondo, R. Nava, J.C. Valdiviezo, G. Cristobal, B. Escalante-Ramirez, M.J. Ruiz-Serrano, J. Pascau and M. Desco, Comparative evaluation of autofocus algorithms for a real-time system for automatic detection of mycobacterium tuberculosis, Cytometry Part A 81A (2012), 213-221.

[19] Y.C. Qiu, X.W. Wang, X.D. Chen, Y.H. Li, H. Liu, S.B. Li and B. Zheng, Automated detection of analyzable metaphase chromosome cells depicted on scanned digital microscopic images, in Proc SPIE 7627 (2010), 7627181-7627189.

[20] H. Xie, W.B. Rong and L.N. Sun, Construction and evaluation of a wavelet-based focus measure for microscopy imaging, Microscopy Research and Technique 70 (2007), 987-995.

[21] Y. Ge and B.J. Nelson, Wavelet-based autofocusing and unsupervised segmentation of microscopic images, in 2003 IEEE/RSJ International Conference on Intelligent Robots and Systems 3 (2003), 2143-2148.

[22] Y.C. Qiu, X.D. Chen, Y.H. Li, B. Zheng, S.B. Li, W.R. Chen and H. Liu, The impact of the optical depth of field on cytogenetic image quality in scanning microscopy, Journal of Biomedical Optics 17 (2012), 0960171-0960177.

[23] Introduction to fourier optics. J.W. Goodman, ed., Roberts and Company Publishers, Colorado, 2004, pp. 145-154.

[24] K. Truong, A. Gibaud, G. Zalcman, M.N. Guilly, M. Antoine, F. Commo, C. Fouquet, J. Cadranel, T. Soussi, B. Dutrillaux and B. Malfoy, Quantitative fish determination of chromosome 3 arm imbalances in lung tumors by automated image cytometry, Medical Science Monitor 10 (2004), 426-432.

[25] B. Kajtar, G. Mehes, T. Lorch, L. Deak, M. Kneifne, D. Alpar and L. Pajor, Automated fluorescent in situ hybridization (fish) analysis of $\mathrm{t}(9 ; 22)(\mathrm{q} 34 ; \mathrm{q} 11)$ in interphase nuclei, Cytometry Part A 69A (2006), 506-514. 
[26] L.J. Van Vliet, I.T. Young and B.H. Mayall, The athena semiautomated karyotyping system, Cytometry 11 (1990), 51-58.

[27] Y.C. Qiu, X.D. Chen, Z. Li, Y.H. Li, W.R. Chen, B. Zheng, S.B. $\mathrm{Li}$ and $\mathrm{H}$. Liu, An automatic scanning method for high throughput microscopic system to facilitate medical genetic diagnosis: An initial study, in Proc of SPIE 8224 (2012), 82240E1-5.

[28] M. Zeder and J. Pernthaler, Multispot live-image autofocusing for high-throughput microscopy of fluorescently stained bacteria, Cytometry Part A 75A (2009), 781-788.
[29] S. Chowdhury, M. Kandhavelu, O. Yli-Harja and A.S. Ribeiro, An interacting multiple model filter-based autofocus strategy for confocal time-lapse microscopy, Journal of Microscopy $\mathbf{2 4 5}$ (2012), 265-275.

[30] M.E. Rudnaya, H.G. ter Morsche, J.M.L. Maubach and R.M.M. Mattheij, A derivative-based fast autofocus method in electron microscopy, Journal of Mathematical Imaging and Vision 44 (2012), 38-51. 


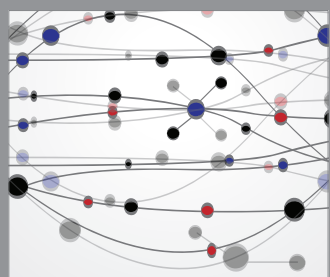

The Scientific World Journal
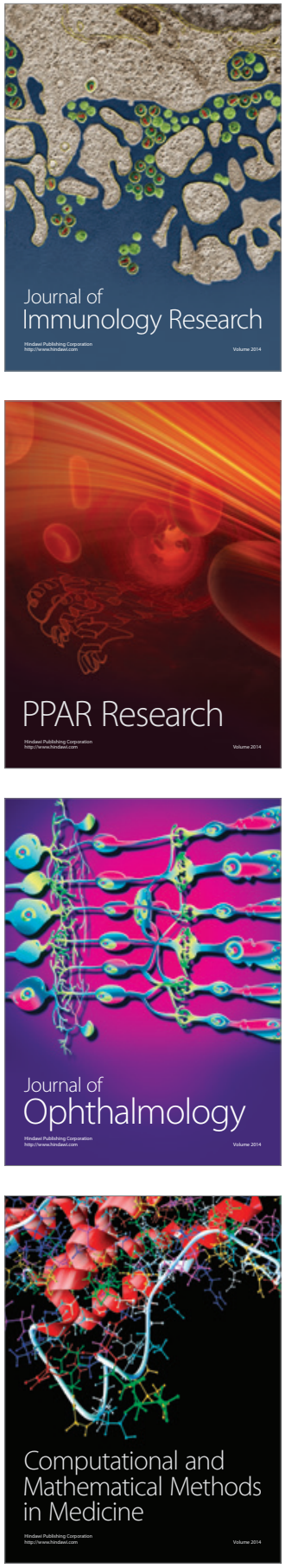

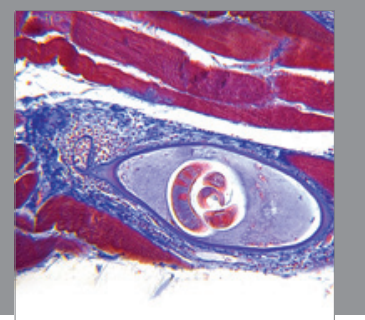

Gastroenterology

Research and Practice
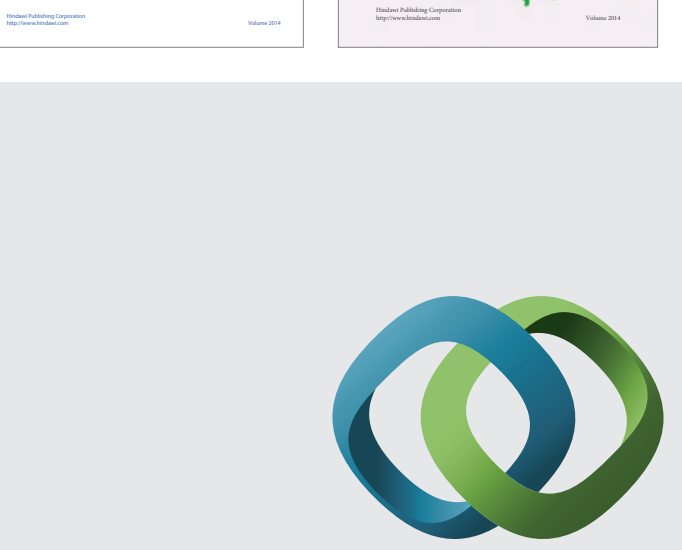

\section{Hindawi}

Submit your manuscripts at

http://www.hindawi.com
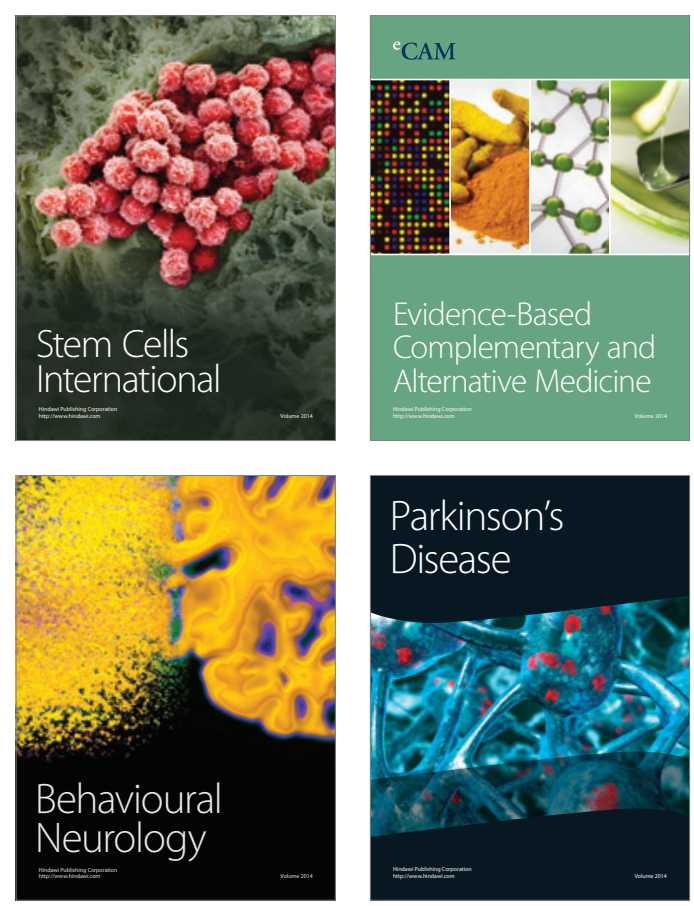

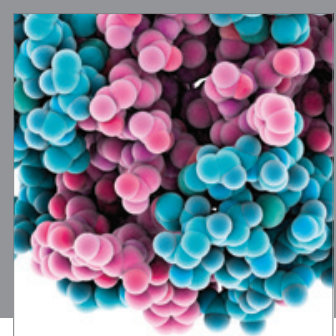

Journal of
Diabetes Research

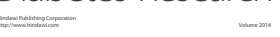

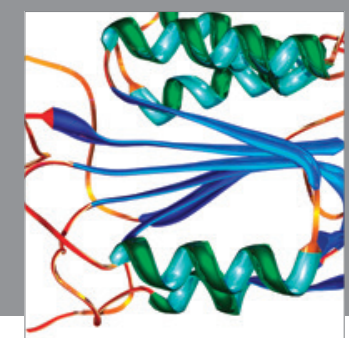

Disease Markers
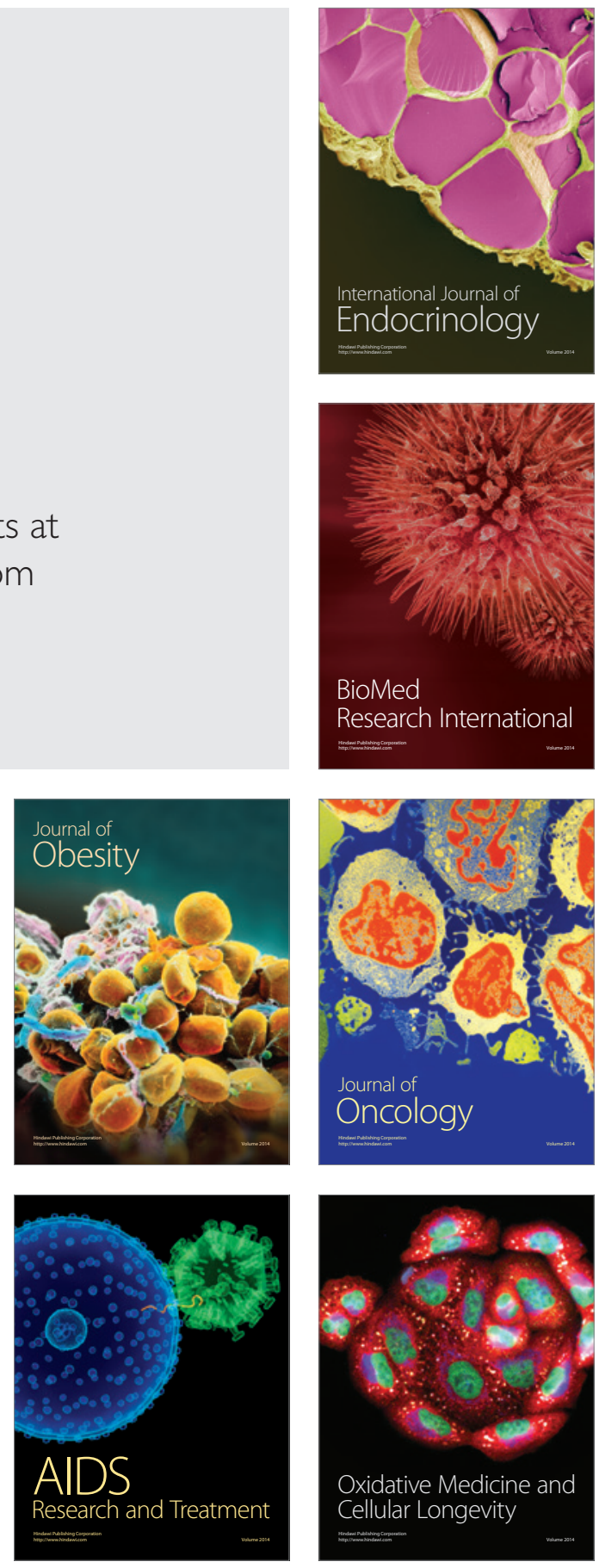